\title{
Ecología trófica de la Garza blanca Ardea alba (Pelecaniformes: Ardeidae) en un humedal del río Paraná, Argentina
}

\author{
Rodrigo Ezequiel Lorenzón ${ }^{1}$, Ana Laura Ronchi Virgolini² y Adolfo Héctor Beltzer ${ }^{1}$ \\ 1. Instituto Nacional de Limnología CONICET-UNL. Ciudad Universitaria s/n, Paraje "El Pozo". CP 3000. Santa Fe, Argentina; \\ rodrigo_lorenzon@hotmail.com, adolfohec2001@yahoo.com.ar \\ 2. Facultad de Ciencia y Tecnología, Universidad Autónoma de Entre Ríos. CP 3108, Diamante, Argentina; Ironchi@gmail.com
}

Recibido 18-V-2012 Corregido 14-IX-2012 Aceptado 8-X-2012

\begin{abstract}
Feeding ecology of Great Egret Ardea alba (Pelecaniformes: Ardeidae) in a wetland of the Paraná River, Argentina. Herons as a group have contributed substantially to the field of evolutionary ecology through studies that have helped elucidate mechanisms that allow coexistence of sympatric species. Detailed descriptions of feeding ecology are essential for understanding mechanisms of coexistence. Despite being one of the most common species in Argentina, detailed studies on the feeding ecology of Great Egrets Ardea alba are scarce. We studied Great Egret feeding ecology through an analysis of the stomach contents of 38 individuals captured between 1990 and 1993 in a wetland on the Paraná River, Argentina. The diet included a total of 33 taxa, with fishes as the predominant prey item. The predominance of fishes in the diet agrees with results of previous studies. There was, however, no evidence of dietary selectivity. The largest proportion of prey $(40 \%)$ were $21-30 \mathrm{~mm}$ in length. Distribution of prey sizes differed between the Great Egret and the sympatric and congeneric Cocoi Heron (Ardea cocoi). Great Egrets primarily foraging in areas characterized by aquatic vegetation and in areas with open water.
\end{abstract}

\section{KEY WORDS}

Ardea alba, Ardeidae, diet, Great Egret, Paraná river, stomach content, wetland.

\section{RESUMEN}

Se estudió la ecología trófica de la Garza blanca (Ardea alba) mediante el análisis del contenido estomacal de 38 individuos capturados entre 1990 y 1993 en un humedal del río Paraná, Argentina. El espectro trófico resultó compuesto por 33 entidades taxonómicas, siendo los peces el ítem - presa predominante. El mayor porcentaje de las presas (40\%) correspondió al intervalo de clase $21-30 \mathrm{~mm}$. La especie no mostró selectividad dietaria y utilizó con mayor frecuencia las unidades ambientales de Vegetación acuática y Aguas abiertas. La predominancia de los peces en la dieta coincidió con lo informado en otros estudios, aunque las características del espectro trófico la señalaron como una especie oportunista. Sólo se encontraron diferencias en el tamaño de las presas al comparar la ecología trófica de la especie con la congenérica y simpátrica Ardea cocoi.

\section{PALABRAS CLAVE}

Ardea alba, Ardeidae, contenido estomacal, río Paraná, humedal.
Los ardeidos constituyen unos de los componentes faunísticos más conspicuos en los humedales en todo el mundo, y al igual que los demás Ciconiiformes poseen varias características que las hacen especialmente adecuadas para estudios ecológicos (Kushlan, 1981). Las distintas especies representan consumidores secundarios de último orden en las tramas tróficas de los humedales. Debido a este papel común en los ecosistemas, junto con las similitudes morfométricas y comportamentales y con la recurrencia de la simpatría entre las especies de la familia (e.g., Ardea alba, Ardea cocoi y Egretta thula en el Neotrópico), los ardeidos han recibido mucha atención en el marco de la ecología evolutiva, lo que se refleja en los estudios dirigidos a dilucidar los mecanismos que permiten la coexistencia de especies (e.g., Ramo \& Busto, 1993; Yanosky et al., 2000; Marín, Guevara \& Bastidas, 2003). 
La información detallada sobre selección de hábitat y distintos aspectos de la historia de vida de las especies, como la ecología trófica, son fundamentales para cualquier estudio dirigido a abordar mecanismos de coexistencia de especies similares. Aunque en el hemisferio norte, estos conocimientos son abundantes (Willard, 1977; Custer \& Osborn, 1978; Kent, 1986), las aves del neotrópico han recibido menos atención (Poulin, Lefebvre \& Mcneil, 1992), y sólo a partir de las últimas dos décadas han sido sujeto de estudios de esta índole (e.g., Ducommun et al., 2008, Ducommun et al., 2010).

Existen 64 especies de ardeidos en el mundo (Clements et al., 2011), de las que 28 se encuentran en el Neotrópico (Remsen et al., 2012). La Garza blanca Ardea alba es una especie cosmopolita, ampliamente distribuida en América, África, India, sudeste de Asia, Papua Nueva Guinea y Australia. A pesar de ser una de las especies más frecuentes en Argentina, los estudios detallados (i.e., estudios que brinden inventarios taxonómicos de ítems tróficos a nivel de familias y especies para muestras representativas de distintas localidades) sobre la ecología alimentaria de esta especie son muy escasos, encontrándose datos aislados y sobre los grandes grupos de organismos que componen su dieta (Zotta, 1934; Beltzer \& Oliveros, 1981).

Al tratarse de una especie cosmopolita, la información comparativa sobre los componentes tróficos en humedales de diferentes ecorregiones a lo largo del área de distribución de la especie puede servir de base para comprender la plasticidad de la especie, o por el contario, la similitud entre diferentes ecosistemas acuáticos a pesar de las variaciones geográficas y climáticas. De este modo, se pone a prueba la hipótesis de que las aves, bajo diferentes condiciones ecológicas en la extensión del área de distribución geográfica, modifican aspectos de la ecología trófica para explotar más eficientemente las condiciones locales. En este marco, el objetivo de este trabajo es aportar datos detallados y susceptibles de comparación sobre la ecología trófica de la Garza blanca ( $A$. alba) en base a individuos capturados en humedales de la planicie de inundación del río Paraná Medio, Argentina. Finalmente, esta información es enmarcada en el conjunto de información existente para la especie en otras latitudes y con respecto a otras especies emparentadas y similares de ardeidos.

\section{METODOLOGÍA}

\section{Área de estudio}

Las capturas se efectuaron en la Isla Carabajal, Provincia de Santa Fe, Argentina ( $31^{\circ} 39^{\prime} \mathrm{S}$ y $60^{\circ} 42^{\prime} \mathrm{W}$ ), isla que forma parte de la planicie de inundación del río Paraná
Medio, incluida en la Ecoregión Delta e Islas del Paraná por Burkart (1999). La Ecoregión comprende ambientes acuáticos permanentes, temporarios y sectores de tierra firme (Neiff, 1999). La Isla Carabajal comprende una superficie estimada en unas 4000 hectáreas, destacándose en ella numerosos cuerpos de agua leníticos, algunos de considerable extensión (hasta 250ha).

Para este estudio, y siguiendo los criterios propuestos por Beltzer (1990, 1991), Neiff (1986) y Beltzer y Neiff (1992) para la planicie de inundación del río Paraná, se diferenciaron las siguientes Unidades de Vegetación y Ambiente (UVAs): Aguas abiertas, Vegetación acuática, Bosque en galería, Bosque, Pajonal, Pastizal y Playa. La Vegetación acuática, tanto flotante como arraigada, ocupa la mayor extensión en el sistema acuático, con variaciones que dependen del ciclo hidrológico (Beltzer \& Neiff, 1992) y entre las especies más frecuentes se encuentran Eichhornia crasippes, E. azurea, Panicum sp., Polygonum sp, Pistia stratiotes, Azolla sp. y Salvinia sp. El Bosque en galería se ubica siguiendo el curso de los ríos y arroyos internos, y sus especies vegetales típicas son: Salix humboltiana y Tessaria integrifolia, propia de albardones incipientes, mientras que en aquellos más viejos se registra la presencia de otras especies como Nectandra falcifolia, Albizia inundata, Sapium haematospermun y algunas trepadoras como Ipomea sp. El Bosque se ubica en los albardones altos y arenosos, siendo Acacia caven la especie dominante. El Pajonal es un ambiente en el que la especie dominante es Panicum prionitis, y se ubica en las áreas de altura intermedia, entre los albardones y las lagunas, al igual que el Pastizal, caracterizado por la presencia de gramíneas bajas. La Playa carece de vegetación, y es de reducida extensión aunque su amplitud depende de los pulsos hidrosedimentológicos.

\section{Análisis de contenidos estomacales}

Se estudiaron 38 estómagos de ejemplares capturados con arma de fuego para fines científicos entre 1990 y 1993 (primavera: ocho estómagos, verano: 11 estómagos, otoño: seis estómagos e invierno: 13 estómagos), los que fueron depositados en la colección de referencia del Instituto Nacional de Limnología (INALI - CONICET - UNL). Se registró la hora de captura y el peso de cada uno de los individuos. A los estómagos se les inyectó una solución de formol al 10\% para detener el proceso digestivo. Además, se realizaron observaciones de campo previas para determinar las UVAs utilizadas para alimentación y las horas de actividad. Los contenidos estomacales fueron luego analizados individualmente en laboratorio, identificándose y cuantificándose los organismos a distintos niveles de resolución taxonómica. Para el conteo de las ingestas en 
avanzado estado de digestión, se consideraron como individuos aquellos que conservaron estructuras o piezas claves para su identificación, tales como cabezas, mandíbulas, élitros y quelíceros.

\section{Análisis de datos}

Se calculó el índice de diversidad de Shannon - Wiener para cada uno de los estómagos analizados (Magurran, 2004). En base al índice de diversidad por estómago se construyó la curva de acumulación de la diversidad trófica para evaluar la representatividad del número de muestras (estómagos) estudiados. Esto se realizó mediante el procedimiento de simulación de Monte Carlo implementado con el programa informático ECOSIM Versión 7 (Gotelli \& Entsminger, 2006).

Con el objeto de establecer la contribución de cada categoría de alimento a la dieta de la especie, se aplicó el índice de importancia relativa (IRI; Pinkas, Oliphant \& Iverson, 1971), que responde a la fórmula $I R I=\% F O(\% \mathrm{~N}+$ $\% \mathrm{~V})$, donde $\% \mathrm{FO}$ es la frecuencia porcentual de la categoría de alimento en los tractos digestivos analizados, \%N es el porcentaje numérico de la categoría de alimento y \%V el porcentaje volumétrico de la misma.Para el cálculo de la amplitud trófica del nicho por estación, se aplicó el índice de Levins (1968): $\mathrm{N}_{\mathrm{B}}=\mathrm{v}\left(\sum \mathrm{Pij}^{2}\right)^{-1}$, donde Pij es la proporción del ítem i en la muestra j. La eficiencia alimentaria, $\mathrm{Pe}$, se estimó para cada estación del año mediante la expresión de Acosta-Cruz, Torres y Mujica-Valdés (1988). La selectividad dietaria se evaluó aplicando la Correlación de Rangos de Spearman (rs, Schefler, 1969; Sokal \& Rohlf ,1979). Para realizar esta correlación se utilizó una estimación cualitativa de la abundancia de las presas en el área de estudio, de acuerdo con consulta a especialistas y observaciones personales. Se establecieron así las siguientes grandes categorías de abundancia de presas: Abundante (1), Común (2), Poco común (3) y Escaso (4). Con el objeto de evaluar la asociación de la especie con las diferentes unidades de ambiente de la planicie de inundación del río Paraná se estimó la preferencia para cada una de las UVAs mediante el indice $P i$ de Duncan (1983): $P i=\log / \mathrm{Vi} /+1$, donde $V i$ es el porcentaje de individuos registrados en cada unidad de ambiente (UVA) y Ai es el porcentaje de cobertura de cada UVA, los que se obtuvieron mediante fotografías aéreas. Siguiendo el criterio propuesto por Bignal, Curtis y Matthews (1988), los valores de este índice superiores a 0,3 indican una alta preferencia por una determinada unidad de ambiente y vegetación, en tanto que valores inferiores señalan una preferencia más baja.

\section{RESULTADOS}

Todos los estómagos analizados contuvieron alimento. El espectro trófico resultó compuesto por 33 entidades taxonómicas, cuyo número y frecuencia se muestran en el Cuadro 1. Los peces fueron el componente más importante en el espectro trófico de la Garza blanca (70\% de las entidades taxonómicas). La curva de acumulación de la diversidad trófica (Fig.1) estuvo próxima a alcanzar una asíntota, indicando que la muestra representó la mayoría de los ítems tróficos de la especie para el área de estudio.

La aplicación del índice de importancia relativa (IRI) señaló que los peces $(\mathrm{IRI}=18,200)$ fueron el componente más importante en cantidad, volumen y frecuencia; siguiéndole los insectos $(\mathrm{IRI}=1273)$ y los arácnidos -crustáceos (IRI=210).

El mayor porcentaje de las presas (40\%) correspondió al intervalo de clase 21-30mm; siendo en su mayoría peces de mediano porte tales como Aphiocarax rubripinnis, Astyanax fasciatus, Serrasalmus spilopleura y Curimatorbis platanus. Los peces y los insectos fueron las ingestas de menor talla, comprendidas en los intervalos 0-10 y 11$20 \mathrm{~mm}$. Un $15 \%$ de las presas correspondió a aquellas que superaron los $40 \mathrm{~mm}$, representadas por juveniles de Salminus maxillosos, Leporinus obtusidens y, dentro de los Decapoda, por Trichodactyllus borelianus (Fig. 2).

Los valores por estación de la amplitud de nicho y la eficiencia alimentaria se muestran en el Cuadro 2. La especie no mostró selectividad dietaria entre los ítems - presa (Spearman, p> 0,001). La especie utilizó todas las unidades ambientales consideradas, con excepción de los pastizales, siendo la vegetación acuática y las aguas abiertas las unidades más frecuentadas (Cuadro 3).

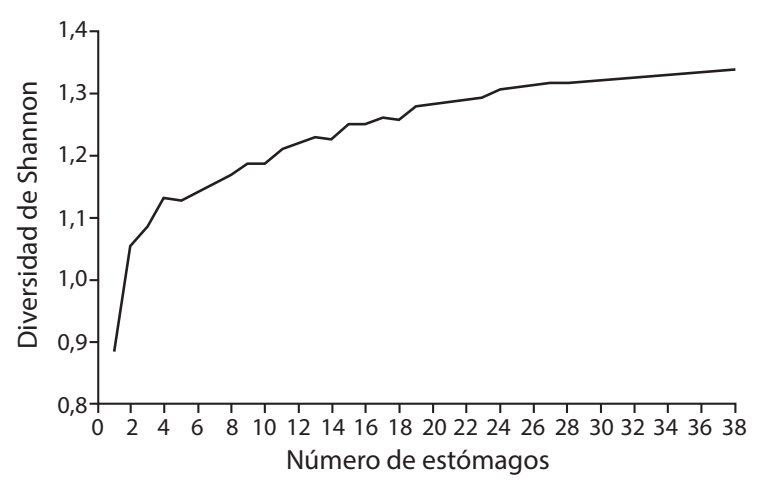

FIG. 1. Curva de acumulación de la diversidad trófica por estómago $(\mathrm{H})$ de la Garza blanca (Ardea alba) en un humedal del río Paraná. 
CUADRO 1

Espectro trófico de la Garza blanca (Ardea alba) en un humedal del río Paraná, Argentina

\begin{tabular}{|c|c|c|}
\hline Organismo & $\mathbf{N}$ & Fo \\
\hline \multicolumn{3}{|l|}{ Pisces } \\
\hline \multicolumn{3}{|l|}{ Characidae } \\
\hline Aphiocarax rubripinnis & 8 & 5 \\
\hline Astyanax fasciatus & 3 & 2 \\
\hline Astyanax sp. & 13 & 9 \\
\hline Salminus maxillosus & 4 & 2 \\
\hline \multicolumn{3}{|l|}{ Serradalmidae } \\
\hline Serrasalmus spilopleura & 1 & 1 \\
\hline \multicolumn{3}{|l|}{ Anostomidae } \\
\hline Leporinus obtusidens & 10 & 7 \\
\hline Schizondon fasciatum & 4 & 2 \\
\hline \multicolumn{3}{|l|}{ Hemiodidae } \\
\hline Appareiodon affinis & 3 & 2 \\
\hline Characidium fasciatum & 1 & 1 \\
\hline \multicolumn{3}{|l|}{ Curimatidae } \\
\hline Prochilodus lineatus & 6 & 3 \\
\hline Curimatorbis platanus & 29 & 18 \\
\hline Gasterostomus latior & 3 & 1 \\
\hline n.i. & 32 & 6 \\
\hline
\end{tabular}

Rhamphichtyidae

Hypopomus brevirostris

Pimelodidae

Pimelodus albicans

n.i.

Callichthyidae

Corydoras hastatus

Corydoras sp.

Hoplosternum thoracatum
CUADRO 1 (Continuación...)

Espectro trófico de la Garza blanca (Ardea alba) en un humedal del río Paraná, Argentina

\begin{tabular}{|c|c|c|}
\hline Organismo & $\mathbf{N}$ & Fo \\
\hline \multicolumn{3}{|l|}{ Cyprinodontidae } \\
\hline Pterolebias longipinnis & 1 & 1 \\
\hline \multicolumn{3}{|l|}{ Cichlidae } \\
\hline Apistogramma combrae & 1 & 1 \\
\hline n.i. & 3 & 3 \\
\hline \multicolumn{3}{|l|}{ Lesbiasinidae } \\
\hline Pyrrhulina australis & 13 & 7 \\
\hline \multicolumn{3}{|l|}{ Insecta } \\
\hline \multicolumn{3}{|l|}{ Hemiptera } \\
\hline \multicolumn{3}{|l|}{ Belostomidae } \\
\hline Belostoma micantulum & 5 & 3 \\
\hline Belostoma sp. & 1 & 1 \\
\hline \multicolumn{3}{|l|}{ Orthoptera } \\
\hline Acrididae n.i. & 1 & 1 \\
\hline \multicolumn{3}{|l|}{ Paulinidae } \\
\hline Paulinia acuminata & 3 & 2 \\
\hline Coleoptera n.i. & 3 & 3 \\
\hline Hymenoptera n.i. & 1 & 1 \\
\hline Aranae n.i. & 2 & 1 \\
\hline
\end{tabular}

Crustacea

Decapoda

Trichodactyllidae

$\begin{array}{lll}\text { Trichidactyllus borelianus } & 1 & 1\end{array}$

Palaeminidae

Machrobrachium borellii $\quad 5 \quad 3$

$\begin{array}{lll}\text { Palaemonetes argentinus } & 1 & 1\end{array}$

$\mathrm{N}=$ número de individuos de cada categoría de alimento $\mathrm{Fo}=$ frecuencia de cada categoría de alimento.

\section{CUADRO 2}

Amplitud del nicho y eficiencia alimentaria por estación de la Garza blanca (Ardea alba) en un humedal del río Paraná, Argentina.

\begin{tabular}{lcccc}
\hline & Primavera & Verano & Otoño & Invierno \\
\hline Amplitud de nicho & 3,86 & 5,57 & 2,94 & 3,95 \\
Eficiencia alimentaria (\%) & 99,31 & 99,11 & 98,80 & 99
\end{tabular}




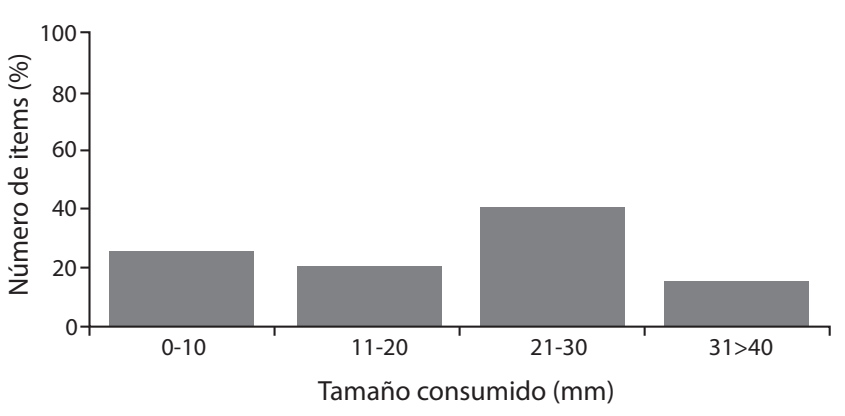

FIG. 2. Porcentaje del número de ítems-presa para cada intervalo de clase de tamaños (en $\mathrm{mm}$ ) consumidos por la Garza blanca (Ardea alba) en un humedal del río Paraná, Argentina.
CUADRO 3

Índice de preferencia (Pi) para cada Unidad de vegetación y ambiente (UVA) de la Garza blanca (Ardea alba) en un humedal del río Paraná, Argentina.

\begin{tabular}{lc}
\multicolumn{1}{c}{ UVA } & Pi \\
Aguas abiertas & 34 \\
Vegetación acuática & 68 \\
Bosque en galería & 11 \\
Pajonal & 18 \\
Pastizal & - \\
Monte & 21 \\
Playa arenosa & 14
\end{tabular}

Además, se ha informado predación sobre roedores y serpientes (Sick, 1997) e incluso aves (Campbell \& Wolf, 1977; Reppening, 1977), aunque esto no ha sido encontrado en el presente estudio. La presencia de un volumen muy bajo de la fracción vegetal, representado mayormente por $R i$ ciocarpus natans, tuvo un elevado porcentaje de aparición en los estómagos analizados, aunque su ingestión fue considerada accidental.

Los valores de amplitud de nicho trófico, los que mostraron diferencias estacionales marcadas, y la ausencia de selectividad, de acuerdo con la no significancia de la prueba de Correlación de Rangos de Spearman, son consistentes con la idea de que la Garza blanca es una especie oportunista. Este patrón es también reportado por Smith (1997), aunque mediante comparaciones interanuales e intercoloniales de los tamaños y tipos de presa. Este autor también sugiere que la Garza blanca forrajea oportunistamente sobre las presas disponibles localmente, cambiando tipos de presa de acuerdo con los cambios en la oferta trófica debidos a variaciones hidrológicos y en las condiciones del hábitat. En el área de estudio, este escenario concuerda con los cambios temporales en la disponibilidad de recursos debido a variaciones del nivel hidrométrico (Beltzer \& Neiff, 1992). Esta flexibilidad es además evidenciada por la diversidad de ítems - presa encontrados en los contenidos estomacales. Finalmente, los valores de eficiencia alimentaria encontrados para la Garza blanca concuerdan con Ricklefs (1998), quien afirma que valores entre 60 y $90 \%$ corresponden a predadores que consumen alimento de origen animal.

Los valores obtenidos del Índice de preferencia de UVAs (Pi) muestran una alta preferencia de la especie por las unidades de vegetación acuática y aguas abiertas. 
Similarmente, Custer y Osborn (1978) observaron que la Garza blanca utiliza áreas vegetadas como sitios de alimentación. Es posible que esto se relacione también al espectro trófico, dado que la Garza blanca, además de atrapar peces en los ambientes acuáticos, muestra un gran porcentaje de insectos en su dieta, los que podrían ser obtenidos de la vegetación acuática. El pastizal fue la única unidad ambiental a la que la Garza blanca no estuvo asociada, al igual que lo informado para la Garza mora (Ducommun et al., 2010), otra especie piscívora, y a diferencia de otras especies primariamente insectivoras como Syrigma sibilatrix y Bubulcus ibis (Beltzer, 2007; Ducommun et al., 2008). Del mismo modo, esta especie, al igual que otras de gran tamaño, como las congenéricas Ardea cocoi, Ardea herodias y Ardea cinerea, tendrían la capacidad de aventurarse en aguas abiertas debido fundamentalmente al gran largo de sus tarsos, aprovechando así humedales más profundos que otras especies.

La especie, al parecer, comparte una dieta fundamentalmente piscívora con la congenérica y simpátrica A. cocoi (Ducommun et al., 2010). No obstante, a diferencia de esta, los insectos representan un ítem alimentario también muy importante (sólo representan un ítem accesorio en A. cocoi, Ducommun et al., 2010). Del mismo modo, estas especies parecerían diferenciarse con respecto a los tamaños de las presas más frecuentemente consumidas, dado que la Garza mora preda más frecuentemente sobre presas de mayor tamaño (entre 150 y $400 \mathrm{~mm}$, Ducommun et al., 2010), lo que concuerda con la idea de una relación directa entre el tamaño de un ave y el de sus presas (Brabata \& Carmona, 1999). Finalmente, los resultados indican una diferenciación en los ritmos diarios de actividad alimentaria, dado que aunque comparten un pico de actividad durante las horas del mediodía, la Garza blanca posee un segundo pico de actividad en horas crepusculares y posiblemente nocturnas, lo que no fue informado en los estudios disponibles sobre la ecología trófica de la Garza mora.

\section{REFERENCIAS}

Acosta Cruz, M., Torres, O. \& Mugica Valdés, L. (1988). Subnicho trófico de Dendrocygna bicolor (Vieillot) (Aves: Anatidae) en dos arroceras de Cuba. Ciencias Biológicas 19/20, 41-50.

Bancroft, G.T., Jewell, S.D. \& Strong, A.M. (1990). Foraging and nesting ecology of herons in the lower Everglades relative to water conditions (Final report). Water Management District, West Palm Beach: South Florida, Florida

Beltzer, A.H. (1990). Biología alimentaria del gavilán común Buteo magnirostris (Aves: Accipitridae) en el valle aluvial del rio Paraná medio, Argentina. Ornitología Neotropical 1, 1-7.
Beltzer, A.H. (1991). Aspects of the foraging ecology of the waders Tringa flavipes, Calidris fuscicollis and Charadrius coIlaris (Aves: Scolpacidae; Charadriidae) in Del Cristal Pond (Santa Fe, Argentine). Studies on Neotropical Fauna and Environment 26, 65-73.

Beltzer, A.H. (2007). Relaciones tróficas y mecanismos de segregación ecológica entre las especies de garzas del valle de inundación del río Paraná (AVES: ARDEIDAE). (Tesis inédita de doctorado). Universidad Nacional de La Plata: La Plata, Argentina.

Beltzer, A.H. \& Oliveros, O.B. (1981). Alimentación de aves en el valle aluvial del río Paraná Medio. II. Egretta alba egretta (Gmelin, 1789) y Egretta thula thula (Molina, 1782) (Ciconiiformes: Ardeidae). Ecología 6, 119-124.

Beltzer, A.H. \& Neiff, J. (1992). Distribución de las aves en el valle del río Paraná. Relación con el régimen pulsátil y la vegetación. Ambiente Subtropical 2, 77-102.

Bignal, E.M., Curtis, D.J. \& Matthews, J.L. (1988). Islau Land Types. Birds habitats and nature conservation. Part. 1: Land use and birds on Islay. Nature Conservancy Council: Peterborough, England.

Brabata, G. \& Carmona, R. (1999). Conducta alimentaria de cuatro especies de aves playeras (Charadriiformes: Scolopacidae) en Chametla, B.C.S., México. Revista de Biología Tropical 47, 239-243.

Burkart, R., Bárbaro, N., Sánchez, R.O. \& Gómez, D. A. (1999). Ecorregiones de la Argentina. PRODIA, APN: Buenos Aires, Argentina.

Campbell, E.G. \& Wolf, G.A. (1977). Great egret predation on a Virginia Rail. Western Birds 8, 64.

Clements, J.F., Schulenberg, T.S., Iliff, M.J., Sullivan, B.L., Wood, C.L. \& Roberson, D. (2011). The Clements checklist of birds of the world: Version 6.6. Recuperado de http://www.birds.cornell.edu/clementschecklist/ downloadable-clements-checklist

Custer, T.W. \& Osborn, R.G. (1978). Feeding habitat use by colonially - breeding Herons, Egrets, and Ibises in North Carolina. Auk 95, 733-743.

Ducommun, M.P., Quiroga, M.A., Beltzer, A.H. \& Schnack, J.A. (2008). Diet of Cattle Egrets in the flood valley of the paraná river, north Argentina. Avian Biology Research 1, 145-151.

Ducommun, M.P., Beltzer, A.H., Ronchi Virgolini, A.L. \& Quiroga, M.Q. (2010). Diet of Cocoi Heron (Ardea cocoi) in the Flood Valley of the Paraná River. Avian Biology Research 3, 115-121.

Duncan, P. (1983). Determination of the use of habitat by horses in Mediterranean wetland. Journal of Ecology Animal 52, 93-109.

Frederick, P.C. (1993). Wading bird nesting success studies in the Water Conservation Areas of the Everglades, 1992 (Final report). South Florida Water Management District: West Palm Beach, Florida. 
Gotelli, N.J. \& Entsminger, G.L. (2006). EcoSim: null models software for ecology, version 7. Vermount: Acquired Intelligence, Inc., and Kesey-Bear: Jericho, Vermont,USA.

Kent, D.M. (1986). Behavior, Habitat Use, and Food of Three Egrets in a Marine Habitat. Colonial Waterbirds 9, 25-30.

Kushlan, J.J. (1981). Resource use strategies for wading birds. Wilson Bulletin 93, 145-163.

Levins, R. (1968). Evolution in changing environment. Princeton University Press: Princeton, New Jersy, USA.

Magurran, A.E. (2004). Measuring Biological Diversity. Blackwell Publishing: Oxford, U.K.

Marín, G.E., Guevara, E.V. \& Bastidas, L.V. (2003). Algunos componentes y aspectos ecológicos de la dieta de aves Ciconiiformes en ecosistemas marino - costeros. Saber, Universidad de Oriente 15, 99-105.

Miranda, L. \& Collazo, J.A. (1997). Food Habits of 4 species of Wading Birds (Ardeidae) in a Tropical Mangrove Swamp. Colonial Waterbirds 20, 413-418.

Moreno, A.B., Lagos, A.R. \& Alves, M.A. (2004). Water depth selection during foraging and efficiency in prey capture by the egrets Casmerodius albus and Egretta thula (Aves, Ardeidae) in a urban lagoon in Rio de Janeiro State, Brazil. Iheringia, Série Zoologia 95, 107-109.

Neiff, J.J. (1986). Las grandes unidades de vegetación y los ambientes insulares del río Paraná en su tramo CandelariaItá Ibaté. Revista de la Asociación de Ciencias Naturales del Litoral 17, 7-30.

Neiff, J.J. (1999). El régimen de los pulsos en ríos y grandes humedales del Sudamérica. En A.I. Malvarez \& P. Kandus (eds.). Tópicos sobre grandes humedales Sudamericanos (pp. 97 146). ORCYT-MAB (UNESCO): Montevideo, Uruguay

Ntiamoa-Baidú, Y., Piersma, T., Wiersma, P., Poot, M., Batley, P. \& Gordon, C. (1998). Habitat selection, daily foraging routines and diet of waterbirds in coatal lagoon in Ghana. Ibis $140,89-103$.

Pinkas, L., Oliphant, M.S. \& Iverson, Z.L. (1971). Food habits of albacore bluefin tuna and bonito in California waters. Dept. Fish Game, Fish Bulletin 152, 1-105.
Poulin, B., Lefebvre, G. \& Mcneil, R. (1992). Tropical avian phenology in relation to abundance and exploitation of food resources. Ecology 73, 2295-2309.

Ramo, C. \& Busto, C. (1993). Resource use by herons in a Yucatán wetland during the breeding season. Wilson Bulletin 105, 573-586.

Remsen, J.V.J., Cadena, C.D., Jaramillo, A., Nores, M., Pacheco, J.F., Pérez-Emán,, J., Robbins, M.B., Stiles, F.G., Stotz, D.F. \& Zimmer, K.J. (2012). A classification of the bird species of South America. Versión Marzo de 2012. American Ornithologists'Union. Recuperado de http://www.museum. Isu.edu/ Remsen/SACCBaseline.html

Reppening, R. (1977). Great Egret preys on sandpiper. Auk 94, 171.

Ricklefs, R.E. (1998). Invitación a la ecología. Economía de la naturaleza. Médica Panamericana: Buenos Aires, Argentina

Schefler, W. (1969). Bioestadística. Fondo Educativo Interamericano S.A. : México D.F., México.

Sick, H. (1977). Ornitologia brasileira. Nova Fronteira: Río de Janeiro, Brasil.

Smith, J.P. (1997). Nesting Season Food Habits of 4 Species of Herons and Egrets at Lake Okeechobee, Florida. Colonial Waterbirds 20, $198-220$.

Sokal, R.R. \& Rohlf, F.J. (1979). Biometría. Principios y métodos estadísticos en la investigación biológica. Blume Ediciones: Madrid, España.

Whitfield, A.K. \& Blaber, S.J. (1979). Feeding ecology of piscivorous birds at Lake St. Lucia, Part 2: wading birds. Ostrich $50,1-9$.

Willard, D.E. (1977). The feeding ecology and behavior of five species of herons in southeastern New Jersey. Condor 79, 462-470.

Yanosky, A.A., Escalante, A., Schuchmann, K. \& Kittlein, M. (2000). Ecología de una Comunidad de Aves Vadeadoras en el Nordeste Argentino. En: E. Cabrera, C. Mercolli \& R. Resquin (eds.). Manejo de Fauna Silvestre en Amazonia y Latinoamérica (pp. 219 - 236). Asunción: Cites-Py, Fundación Moisés Bertoni., Universidad de Florida, Florida, EEUU.

Zotta, A. (1934). Sobre el contenido estomacal de algunas aves. Hornero 5, 376-383. 
